\title{
CARACTERIZAÇÃo FÍSICA E QUÍMICA DE GELÉIA DE AMORA-PRETA ${ }^{1}$
}

\author{
Renata Vieira da MOTA ${ }^{2, *}$
}

\begin{abstract}
RESUMO
A amora-preta é um fruto de alta perecibilidade e por isso seu aproveitamento é preferencialmente industrial. Entretanto, há pouca informação na literatura a respeito do efeito do processamento e do armazenamento na composição química de produtos elaborados a partir destes frutos. Neste trabalho, foram preparadas geléias a partir de sete cultivares de amoreira-preta introduzidas em Minas Gerais, pela EPAMIG. As geléias foram avaliadas após o processamento e armazenamento por 40 e 90 dias à temperatura ambiente quanto ao $\mathrm{pH}$, sólidos solúveis totais, acidez total titulável, umidade, carboidratos solúveis e antocianinas totais. O aquecimento da polpa na elaboração da geléia resultou em uma redução de $8,8 \%$ no teor inicial de antocianinas totais. O armazenamento também resultou em perda de antocianinas, em média $32 \%$ nos primeiros 40 dias, com redução para $11 \%$ nos 50 dias subseqüentes. Os teores de antocianinas totais obtidos na geléia variaram de 98,58 mg/100 g (Seleção 97) a 170,66 mg/100 g (Guarani), o que caracteriza este produto como um alimento rico em compostos fenólicos.

Palavras-chave: Rubus sp., cultivares, geléia, armazenamento, composição, antocianinas.
\end{abstract}

\section{SUMMARY}

PHYSICO AND CHEMICAL CHARACTERISATION OF BLACKBERRY JAM. Blackberries have a short shelf-life and thus are used mainly as raw material for the food industry. In spite of the wide use of blackberries as processed products, not much is known about the effects of processing and storage concerning the chemical composition of such products. In this paper, jams were prepared from seven blackberry cultivars grown in Brazil. Jams were evaluated after processing and storage for 40 and 90 days at room temperature for some parameters related to quality such as the $\mathrm{pH}$, total soluble solids, total titratable acidity, humidity, soluble carbohydrates and total anthocyanin content. The heat applied to blackberry jam products reduced the anthocyanin content at an average of $8.8 \%$. Storage time also reduced the anthocyanin content at an average of $32 \%$ after 40 days at room temperature decreasing to $11 \%$ in the next 50 days of storage. The anthocyanin content obtained in blackberry jams ranged from $98.58 \mathrm{mg} / 100 \mathrm{~g}$ ('97 selection) to $170.66 \mathrm{mg} / 100 \mathrm{~g}$ (Guarani), which characterizes this food as a rich source for phenolic compounds.

Keywords: Rubus sp., cultivars, jam, storage, composition, anthocyanins.

\section{1 - INTRODUÇÃO}

A amoreira-preta (Rubus sp.) é uma espécie arbustiva de porte ereto ou rasteiro, que produz frutos agregados com cerca de quatro a sete gramas, de coloração negra e sabor ácido a doce-ácido. É uma planta rústica que apresenta baixo custo de produção, facilidade de manejo, requer pouca utilização de defensivos agrícolas, sendo, por isso, uma alternativa interessante para cultivo na agricultura familiar [1].

O fruto in natura é altamente nutritivo e contém $85 \%$ de água, $10 \%$ de carboidratos, elevado conteúdo de minerais, vitaminas do complexo B e A e cálcio, além de ser fonte de compostos funcionais, como ácido elágico e antocianinas [3, 19]. Estudos realizados por WANG \& LIN [22] em frutos e folhas de amora-preta, framboesa e morango, indicaram que os frutos maduros de framboesa preta e amora-preta constituem fonte rica em antocianinas (197,2 mg/100 g e $152,8 \mathrm{mg} / 100 \mathrm{~g}$, respectivamente) quando comparados com frutos maduros de framboesa vermelha $(68,0 \mathrm{mg} / 100 \mathrm{~g})$ ou morango (31,9 mg/100 g).

${ }^{1}$ Recebido para publicação em 26/1/2005. Aceito para publicação em 6/7/2006 (001474)

${ }^{2}$ (EPAMIG) Estação Experimental de Caldas,

Av. Santa Cruz, 500, C. P. 33, CEP 37780-000, Caldas (MG), Brasil,

E-mail:renata@epamigcaldas.gov.br

* A quem a correspondência deve ser enviada
Notícias divulgadas pela imprensa a respeito da importância do consumo de alimentos ricos em compostos fenólicos com atividade antioxidante [10, 13] despertaram o interesse dos consumidores por estes produtos.

Entretanto, por apresentar estrutura frágil e alta atividade respiratória, a conservação pós-colheita da amora-preta é relativamente curta. Segundo ANTUNES, DUARTE FILHO \& SOUZA [2], frutos das cultivares Brazos e Comanche conservaram-se em ambiente refrigerado $\left(2{ }^{\circ} \mathrm{C}\right)$ por até nove dias, com redução nos teores de acidez total titulável e de sólidos solúveis totais.

Em razão da produção concentrada nos meses de novembro a fevereiro e a rápida perda de qualidade pós-colheita, há uma grande limitação quanto ao fornecimento dos frutos ao mercado in natura. Uma alternativa viável para o aproveitamento econômico desses frutos é a industrialização, pois estes podem ser congelados, enlatados, processados na forma de polpa para utilização em produtos lácteos (como matéria prima ou aditivo de cor e sabor), ou na forma de sucos e geléias [1].

Entretanto, o processamento e o armazenamento podem afetar a composição dos frutos e, conseqüentemente, suas propriedades benéficas à saúde.

Devido ao crescente interesse em produtos a base de pequenas frutas, este trabalho teve como objetivo avaliar 
a qualidade física e química de geléias elaboradas a partir de sete cultivares de amora-preta.

\section{2 - MATERIAL E MÉTODOS}

Frutos das cultivares Brazos, Tupy, Guarani, Caingangue, Comanche, Cherokee e Seleção 97 foram obtidos no banco de germoplasma da Estação Experimental da EPAMIG (FECD) em Caldas, Minas Gerais.

Logo após a colheita, os frutos foram lavados em água clorada, contendo 25 ppm de cloro residual livre, e selecionados quanto à sanidade. Em seguida, foram distribuídos em bandejas e transferidos para freezer doméstico $\left(-18{ }^{\circ} \mathrm{C}\right)$ para congelamento. Os frutos congelados foram colocados em embalagens plásticas transparentes com fecho hermético e mantidos a $-18{ }^{\circ} \mathrm{C}$ até o processamento.

Os frutos da amoreira-preta não amadurecem ao mesmo tempo e, por isso, devem ser colhidos de forma escalonada durante um período de dois meses e meio a três meses, conforme a cultivar. Para evitar o efeito da época de colheita (início, meio, final de safra) na avaliação da qualidade física e química, uma unidade amostral consistiu de um número igual de frutos obtidos de cada colheita.

Para a elaboração da polpa, os frutos foram mantidos à temperatura ambiente até o descongelamento, sendo, em seguida, triturados em liquidificador por um minuto e peneirados para remoção das sementes. Uma parte da polpa foi separada para a avaliação do pH [15] e teor de antocianinas [17, 20, 21].

Segundo CETEC [6], o pH ótimo para a formação do gel é de 3,0 a 3,2. Como os valores de $\mathrm{pH}$ obtidos para a polpa ficaram na faixa de 3,2 a 3,4, dependendo da cultivar, não foi necessária a adição de agente acidificante na formulação da geléia.

Foram testadas as dosagens de açúcar a 50\% e $75 \%$ do peso da polpa sem adição de pectina. O produto obtido com $75 \%$ de açúcar ficou mais resistente, porém o excesso de açúcar descaracterizou o sabor acidificado da fruta. Assim, a composição das geléias consistiu em aproximadamente 700 g de polpa e sacarose na proporção de 50\%, com substituição de $10 \%$ deste teor por xarope de glicose.

A cocção foi feita em tacho aberto, seguindo o fluxograma proposto por CETEC [6]. A polpa foi aquecida até aproximadamente $70{ }^{\circ} \mathrm{C}$ para a adição da sacarose. Próximo ao ponto final de cozimento, foi acrescentado o xarope de glicose. O ponto final foi determinado quando uma gota da geléia alcançou o fundo de um copo com água fria sem desintegrar. A geléia foi imediatamente transferida para potes de vidro com tampas metálicas com capacidade de $250 \mathrm{~mL}$. Os vidros foram fechados, invertidos e, em seguida, resfriados em água até aproximadamente $40{ }^{\circ} \mathrm{C}$.

As análises físicas e químicas foram realizadas um dia após o processamento e armazenamento por 40 e 90 dias sob condições ambiente de temperatura $\left(20 \pm 2{ }^{\circ} \mathrm{C}\right)$ e luminosidade.
As análises físicas e químicas consistiram na determinação de pH, sólidos solúveis totais (SST), acidez total titulável (ATT), umidade [15], carboidratos [8] e antocianinas totais $[17,20,21]$. A extração das antocianinas foi feita em metanol acidificado com HCL $1 \%$ e o conteúdo estimado como cianidina-3-glucosideo a $532 \mathrm{~nm}$, usando um coeficiente de

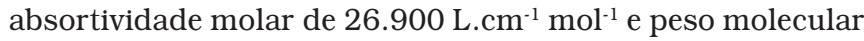
de 449,2 g/mol. O rendimento em geléia foi determinado com base no peso da polpa antes e após o processamento.

Todas as determinações foram efetuadas em triplicata e os resultados submetidos à análise estatística pelo Programa ESTAT [9].

\section{3 - RESULTADOS E DISCUSSÃO}

As geléias apresentaram consistência firme, porém macia, ao serem manuseadas, coloração roxo brilhante, sabor e aroma característicos da amora-preta. Como foram elaboradas de forma caseira, com a determinação do ponto final de cozimento estimado pelo teste da colher [6], as geléias das diferentes cultivares não apresentaram textura uniforme.

O rendimento médio (considerando todos os cultivares) ficou em torno de 96\% (Tabela 1).

O pH das geléias manteve-se na faixa de 3,2 a 3,4, valores próximos aos observados na polpa dos frutos antes do processamento.

O teor de sólidos solúveis das geléias das sete cultivares foi de $50{ }^{\circ} \mathrm{Brix}$ em média, destacando-se o produto obtido da cultivar Brazos (58,03 ${ }^{\circ}$ Brix). Esta geléia também apresentou maior acidez $(1,79 \%)$ e carboidratos solúveis $(51,77 \%)$. Estes resultados parecem estar relacionados à maior concentração da geléia, como pode ser observado pelo baixo rendimento obtido após o processamento $(78,87 \%)$ e pela relação SST/ATT $(32,42)$, dentro da faixa observada para geléias elaboradas a partir das demais cultivares, que variou de 30,50 na cultivar Comanche a 38,65 na cultivar Caingangue (Tabela 1).

A determinação da umidade pela metodologia adotada (secagem em estufa a $105^{\circ} \mathrm{C}$ até peso constante) não é adequada a este tipo de produto, devido à caramelização do açúcar. Os resultados obtidos indicam um teor aproximado de umidade no produto final.

Os valores de antocianinas totais encontrados na polpa dos frutos das sete cultivares de amora avaliadas (110,21 a $194,59 \mathrm{mg} / 100 \mathrm{~g}$ ) estão próximos aos valores obtidos por HASSIMOTTO et al. [14].

O processamento reduziu o teor inicial de antocianinas na maioria das cultivares avaliadas. A geléia apresentou teores de antocianinas totais em média 8,8\% inferiores aos encontrados na polpa (Tabela 1). Presença de oxigênio, ácido ascórbico, hidroximetilfurfural, dióxido de enxofre (utilizado no processo de refino do açúcar) e o aquecimento são fatores que podem ter atuado na degradação deste pigmento [4]. MARKAKIS, LIVINGSTON \& FELLERS [18] estudaram a degradação das antocianinas e verificaram 
TABELA 1 - Características físicas e químicas de geléias elaboradas a partir de sete cultivares de amora-preta.

\begin{tabular}{|c|c|c|c|c|c|c|c|c|c|}
\hline Cultivar & $\begin{array}{c}\text { Rendimento } \\
(\%)\end{array}$ & $\mathrm{pH}^{*}$ & $\begin{array}{l}\mathrm{SST}^{*} \\
\text { ('Brix) }\end{array}$ & $\begin{array}{c}\text { Acidez }^{*} \\
\text { (\% ácido cítrico) }\end{array}$ & $\begin{array}{l}\text { Relação } \\
\text { SST/ATT }\end{array}$ & $\begin{array}{c}\text { Carboidratos* } \\
(\%)\end{array}$ & $\begin{array}{c}\text { Antocianinas* } \\
\text { Geléia }\end{array}$ & $\begin{array}{c}(\mathrm{mg} / 100 \mathrm{~g}) \\
\text { Polpa }\end{array}$ & $\begin{array}{c}\text { Umidade* } \\
(\%)\end{array}$ \\
\hline Caingangue & 99,04 & $\begin{array}{l}3,47 \pm \\
0,01 \mathrm{~A}\end{array}$ & $\begin{array}{l}47,15 \pm \\
0,74 \mathrm{D} \\
\end{array}$ & $\begin{array}{l}1,22 \pm \\
0,02 \mathrm{D}\end{array}$ & 38,65 & $\begin{array}{l}44,56 \pm \\
0,04 \mathrm{~B}\end{array}$ & $\begin{array}{l}115,28 \pm \\
0,74 \mathrm{C} \mathrm{b \#}\end{array}$ & $\begin{array}{l}125,62 \pm \\
0,80 \mathrm{BC} \text { a }\end{array}$ & $\begin{array}{l}45,23 \pm \\
1,34 \mathrm{~B} \\
\end{array}$ \\
\hline Guarani & 95,74 & $\begin{array}{l}3,38 \pm \\
0,00 \mathrm{C}\end{array}$ & $\begin{array}{l}1,59 \pm \\
0,34 \mathrm{~B}\end{array}$ & $\begin{array}{l}1,54 \pm \\
0,02 \mathrm{~B}\end{array}$ & 33,50 & $\begin{array}{l}46,79 \pm \\
2,01 \mathrm{AB}\end{array}$ & $\begin{array}{l}170,66 \pm \\
4,58 \mathrm{~A} \mathrm{~b}\end{array}$ & $\begin{array}{l}194,59 \pm \\
4,72 \mathrm{Aa}\end{array}$ & $\begin{array}{l}46,44 \pm \\
0,62 \mathrm{AB}\end{array}$ \\
\hline Comanche & 98,76 & $\begin{array}{l}3,34 \pm \\
0,00 \mathrm{D}\end{array}$ & $\begin{array}{l}47,28 \pm \\
0,27 \mathrm{D}\end{array}$ & $\begin{array}{l}1,55 \pm \\
0,05 \mathrm{~B}\end{array}$ & 30,50 & $\begin{array}{l}45,77 \pm \\
3,08 \mathrm{AB}\end{array}$ & $\begin{array}{l}110,06 \pm \\
0,95 \mathrm{CD} b\end{array}$ & $\begin{array}{l}121,00 \pm \\
1,04 \mathrm{CD} a\end{array}$ & $\begin{array}{l}45,78 \pm \\
0,33 \mathrm{~B}\end{array}$ \\
\hline Cherokee & 97,79 & $\begin{array}{l}3,39 \pm \\
0,01 \mathrm{BC}\end{array}$ & $\begin{array}{l}49,65 \pm \\
0,69 \mathrm{BC}\end{array}$ & $\begin{array}{l}1,34 \pm \\
0,02 \mathrm{C}\end{array}$ & 37,05 & $\begin{array}{l}47,22 \pm \\
2,04 \mathrm{AB}\end{array}$ & $\begin{array}{l}107,39 \pm \\
1,40 \mathrm{Da}\end{array}$ & $\begin{array}{l}110,21 \pm \\
0,92 \mathrm{E} a\end{array}$ & $\begin{array}{l}42,84 \pm \\
0,45 \mathrm{~B}\end{array}$ \\
\hline Seleção 97 & 103,52 & $\begin{array}{l}3,26 \pm \\
0,01 \mathrm{E}\end{array}$ & $\begin{array}{l}48,31 \pm \\
0,27 \mathrm{CD}\end{array}$ & $\begin{array}{l}1,37 \pm \\
0,02 \mathrm{C}\end{array}$ & 35,26 & $\begin{array}{l}41,96 \pm \\
3,11 \mathrm{~B}\end{array}$ & $\begin{array}{l}98,58 \pm \\
1,54 \mathrm{~Eb}\end{array}$ & $\begin{array}{l}116,46 \pm \\
2,50 \mathrm{DE} \text { a }\end{array}$ & $\begin{array}{l}49,82 \pm \\
2,89 \mathrm{~A}\end{array}$ \\
\hline Tupy & 96,53 & $\begin{array}{l}3,26 \pm \\
0,00 \mathrm{E}\end{array}$ & $\begin{array}{l}48,25 \pm \\
0,09 \mathrm{CD}\end{array}$ & $\begin{array}{l}1,41 \pm \\
0,01 \mathrm{C}\end{array}$ & 34,22 & $\begin{array}{l}46,34 \pm \\
1,61 \mathrm{AB} \\
\end{array}$ & $\begin{array}{l}100,43 \pm \\
0,89 \mathrm{E} \mathrm{b}\end{array}$ & $\begin{array}{l}116,76 \pm \\
1,70 \mathrm{DE} a\end{array}$ & $\begin{array}{l}44,50 \pm \\
0,37 \mathrm{~B}\end{array}$ \\
\hline Brazos & 78,87 & $\begin{array}{l}3,41 \pm \\
0,01 \mathrm{~B}\end{array}$ & $\begin{array}{l}58,03 \pm \\
1,57 \mathrm{~A}\end{array}$ & $\begin{array}{l}1,79 \pm \\
0,04 \mathrm{~A}\end{array}$ & 32,42 & $\begin{array}{l}51,77 \pm \\
2,64 \mathrm{~A}\end{array}$ & $\begin{array}{l}133,76 \pm \\
1,35 \mathrm{~B} \mathrm{a}\end{array}$ & $\begin{array}{l}133,33 \pm \\
3,28 \mathrm{~B} \mathrm{a}\end{array}$ & $\begin{array}{l}43,22 \pm \\
1,01 \mathrm{~B}\end{array}$ \\
\hline
\end{tabular}

*Média de três repetições analíticas \pm desvio padrão. Letras maiúsculas diferentes na mesma coluna indicam diferenças estatísticas significativas entre as cultivares (p < 0,05 ); $\mathrm{e}$ \#Letras minúsculas diferentes na mesma linha indicam diferenças estatísticas significativas entre o teor de antocianinas na polpa e na geléia de cada cultivar ( $<$ 0,05).

efeito deletério do oxigênio, ácido ascórbico, aquecimento e hidroximetilfurfural na retenção do pigmento em suco de morango.

O elevado teor de antocianinas totais observado na geléia da cultivar Brazos (Tabela 1) deve ser resultado da maior concentração dessa geléia quando comparada às demais, uma vez que a formulação foi a mesma para todas as amostras. O teor em ácido ascórbico também pode influenciar na degradação das antocianinas [4, 18]. Resultados obtidos por HASSIMOTTO et al. [14] indicam que, das cultivares avaliadas, Brazos foi a que apresentou os menores teores, em média 9,86 mg/100 g.

As geléias foram avaliadas após 40 e 90 dias de armazenamento em condições ambiente de luminosidade e temperatura. A Tabela 2 ilustra o efeito do armazenamento nos parâmetros mensurados.

Observou-se tendência a aumento no pH e redução nos teores de acidez total titulável e sólidos solúveis totais com o decorrer do armazenamento das geléias. O teor de antocianinas decresceu de forma acentuada (em média 32\%) nos primeiros 40 dias de armazenamento. Nos 50 dias subseqüentes

TABELA 2 - Efeito do armazenamento em condições ambiente por 0, 40 e 90 dias na qualidade da geléia de amora-preta preparada a partir de sete cultivares.

\begin{tabular}{|c|c|c|c|c|c|c|c|}
\hline Cultivar & $\begin{array}{c}\text { Armaz. } \\
\text { (dias) }\end{array}$ & $\mathrm{pH}^{*}$ & $\begin{array}{l}\text { SST* }^{*} \\
\left({ }^{\circ} \text { Brix) }\right.\end{array}$ & $\begin{array}{c}\text { Acidez }^{*} \\
\text { (\% ác. cítrico) }\end{array}$ & $\begin{array}{c}\text { Carboidratos* } \\
(\%)\end{array}$ & $\begin{array}{c}\text { Antocianinas* } \\
(\mathrm{mg} / 100 \mathrm{~g})\end{array}$ & $\begin{array}{c}\text { Umidade }^{*} \\
(\%)\end{array}$ \\
\hline Caingangue & 40 & $3,57 \pm 0,00 \mathrm{~A}$ & $44,37 \pm 0,25 \mathrm{~B}$ & $1,11 \pm 0,02 \mathrm{~B}$ & $46,84 \pm 1,94 \mathrm{~A}$ & $79,80 \pm 5,39 \mathrm{~B}$ & $48,50 \pm 0,08 \mathrm{~A}$ \\
\hline \multirow[t]{3}{*}{ Guarani } & 0 & $3,38 \pm 0,00 \mathrm{~A}$ & $51,59 \pm 0,34 \mathrm{~A}$ & $1,54 \pm 0,02 \mathrm{~A}$ & $46,79 \pm 2,01 \mathrm{~A}$ & $170,66 \pm 4,58 \mathrm{~A}$ & $46,44 \pm 0,62 \mathrm{~A}$ \\
\hline & 40 & $3,40 \pm 0,00 \mathrm{~A}$ & $48,70 \pm 0,04 \mathrm{~B}$ & $1,49 \pm 0,02 \mathrm{~A}$ & $49,55 \pm 2,85 \mathrm{~A}$ & $99,10 \pm 5,70 \mathrm{~B}$ & $46,76 \pm 0,40 \mathrm{~A}$ \\
\hline & 90 & $3,34 \pm 0,05 \mathrm{~A}$ & $45,54 \pm 0,08 \mathrm{C}$ & $1,40 \pm 0,02 \mathrm{~B}$ & $45,91 \pm 2,51 \mathrm{~A}$ & $97,48 \pm 1,31 \mathrm{~B}$ & $45,07 \pm 0,24 \mathrm{~B}$ \\
\hline \multirow{2}{*}{ Comanche } & 40 & $3,35 \pm 0,00 \mathrm{~B}$ & $45,09 \pm 0,09 \mathrm{~B}$ & $1,39 \pm 0,00 \mathrm{~B}$ & $43,11 \pm 3,11 \mathrm{~A}$ & $76,41 \pm 1,76 \mathrm{~B}$ & $49,49 \pm 1,14 \mathrm{~A}$ \\
\hline & 90 & $3,40 \pm 0,00 \mathrm{~A}$ & $44,00 \pm 0,33 \mathrm{C}$ & $1,32 \pm 0,01 \mathrm{C}$ & $42,64 \pm 2,00 \mathrm{~A}$ & $70,24 \pm 1,29 \mathrm{C}$ & $48,89 \pm 0,59 \mathrm{~A}$ \\
\hline \multirow[t]{3}{*}{ Cherokee } & 0 & $3,39 \pm 0,01 \mathrm{~B}$ & $49,65 \pm 0,69 \mathrm{~A}$ & $1,34 \pm 0,02 \mathrm{~A}$ & $47,22 \pm 2,04 \mathrm{~A}$ & $107,38 \pm 1,40 \mathrm{~A}$ & $42,84 \pm 0,45 \mathrm{C}$ \\
\hline & 40 & $3,45 \pm 0,01 \mathrm{~A}$ & $46,60 \pm 0,13 \mathrm{~B}$ & $1,17 \pm 0,01 \mathrm{~B}$ & $46,54 \pm 2,80 \mathrm{~A}$ & $78,35 \pm 2,01 \mathrm{~B}$ & $46,65 \pm 0,31 \mathrm{~A}$ \\
\hline & 90 & $3,44 \pm 0,02 \mathrm{~A}$ & $46,26 \pm 0,19 \mathrm{~B}$ & $1,10 \pm 0,02 \mathrm{C}$ & $49,15 \pm 2,75 \mathrm{~A}$ & $67,27 \pm 2,82 \mathrm{C}$ & $44,33 \pm 0,34 \mathrm{~B}$ \\
\hline Seleção 97 & 0 & $3,26 \pm 0,01 \mathrm{C}$ & $48,31 \pm 0,27 \mathrm{~A}$ & $1,37 \pm 0,02 \mathrm{~A}$ & $41,96 \pm 3,11 \mathrm{~A}$ & $98,58 \pm 1,54 \mathrm{~A}$ & $49,82 \pm 2,89 \mathrm{~A}$ \\
\hline \multirow{2}{*}{ Tupy } & 40 & $3,29 \pm 0,00 \mathrm{~B}$ & $45,11 \pm 0,47 \mathrm{~B}$ & $1,28 \pm 0,03 \mathrm{~B}$ & $46,02 \pm 1,43 \mathrm{~A}$ & $78,74 \pm 2,66 \mathrm{~B}$ & $48,21 \pm 0,40 \mathrm{~A}$ \\
\hline & 90 & $3,35 \pm 0,01 \mathrm{~A}$ & $44,24 \pm 0,71 \mathrm{~B}$ & $1,23 \pm 0,00 \mathrm{C}$ & $43,60 \pm 2,81 \mathrm{~A}$ & $62,48 \pm 3,37 \mathrm{C}$ & $47,33 \pm 0,33 \mathrm{~A}$ \\
\hline \multirow[t]{3}{*}{ Brazos } & 0 & $3,41 \pm 0,01 \mathrm{AB}$ & $58,03 \pm 1,57 \mathrm{~A}$ & $1,79 \pm 0,04 \mathrm{~A}$ & $51,77 \pm 2,64 \mathrm{~A}$ & $133,76 \pm 1,35 \mathrm{~A}$ & $43,22 \pm 1,01 \mathrm{~B}$ \\
\hline & 40 & $3,44 \pm 0,01 \mathrm{~A}$ & $50,74 \pm 0,28 \mathrm{~B}$ & $1,68 \pm 0,01 \mathrm{~B}$ & $49,21 \pm 2,23 \mathrm{~A}$ & $72,57 \pm 0,97 \mathrm{~B}$ & $45,61 \pm 0,22 \mathrm{~A}$ \\
\hline & 90 & $3,38 \pm 0,02 \mathrm{~B}$ & $49,52 \pm 0,26 \mathrm{~B}$ & $1,62 \pm 0,02 \mathrm{~B}$ & $44,64 \pm 3,79 \mathrm{~A}$ & $65,19 \pm 2,23 \mathrm{C}$ & $42,32 \pm 0,20 \mathrm{~B}$ \\
\hline
\end{tabular}


(dos 40 aos 90 dias), a taxa de degradação das antocianinas foi menos acentuada, em média 11\% (Tabela 2 ).

Resultado semelhante foi obtido para a geléia de framboesa. ZAFRILLA, FERRERES \& TOMÁS-BARBERÁN [23] verificaram que o conteúdo de flavonóides da polpa de framboesa decresceu ligeiramente com o processamento para elaboração de geléia e de forma mais acentuada durante o armazenamento.

A degradação da cor durante o armazenamento de geléias também foi observada por FREEDMAN \& FRANCIS [11], CARDOSO et al. [5] e GARCIA-ALONSO et al. [12].

FREEDMAN \& FRANCIS [11] avaliaram o efeito da adição de ácido ascórbico na cor de geléias de morango, amorapreta, maçã e laranja armazenadas por até 32 semanas à temperatura ambiente $\left(22 \pm 2{ }^{\circ} \mathrm{C}\right)$. Os autores observaram degradação da cor das geléias de morango e amora-preta nas primeiras oito semanas de armazenamento, que foi acentuada na presença do ácido ascórbico. De acordo com os autores, a maior descoloração observada nas amostras tratadas com ácido ascórbico deve ser decorrente da combinação do efeito antioxidante do ácido ascórbico e da degradação do pigmento antociânico na presença deste ácido. As mudanças de cor observadas na geléia de amora-preta foram menos acentuadas quando comparadas à geléia de morango. Neste caso, pequenas degradações do pigmento resultam em maior impacto visual do que em um sistema com alta concentração de antocianinas, como a geléia de amora-preta.

CARDOSO et al. [5] observaram decréscimo da cor vermelha ao longo do tempo de armazenamento da geléia de jambo, com influência direta da luz e temperatura. Resultados semelhantes foram observados por GARCIA-ALONSO et al. [12] em um produto à base de suco concentrado de uva, amora-preta, framboesa, cereja e groselha preta. Segundo os autores, as condições de armazenamento afetaram a cor e as concentrações dos compostos antioxidantes, especialmente nas amostras armazenadas a $30{ }^{\circ} \mathrm{C}$, embora a atividade antioxidante total tenha permanecido praticamente inalterada.

Apesar da redução no teor de antocianinas nas geléias, causada pela ação direta do processamento (Tabela 1) ou do armazenamento (Tabela 2), a geléia de amora-preta retém boa parte das antocianinas encontradas na polpa e é uma fonte a ser considerada destes compostos, pois os teores presentes neste produto são superiores aos encontrados nos frutos in natura de outras frutas vermelhas, como morango (30 a $60 \mathrm{mg} / 100 \mathrm{~g}$ ) [7], pitanga roxa (16,23 mg/100 g) [16] ou acerola (3,79 a 59,74 mg/100 g) [17].

\section{4 - CONCLUSÕES}

O fruto da amoreira-preta apresenta bom potencial para elaboração de geléias. O processamento é simples, não requer adição de pectina ou acidificação da polpa.

$\mathrm{O}$ rendimento médio obtido para o produto elaborado com adição de $50 \%$ de açúcar foi de $96 \%$.
O processamento na forma de geléia reduz o teor de antocianinas, porém o produto pode ser considerado como fonte deste composto devido aos elevados teores observados, mesmo após três meses de armazenamento na presença de luz e temperatura média de $20{ }^{\circ} \mathrm{C}$. Entretanto, estudos devem ser realizados para verificar o efeito do processamento e do armazenamento na atividade antioxidante das antocianinas.

Das cultivares avaliadas, Guarani apresentou os maiores níveis de antocianinas totais na geléia (170,66 mg/100 g) e os menores foram observados na cultivar Seleção 97 (98,58 mg/100 g).

\section{5 - REFERÊNCIAS BIBLIOGRÁFICAS}

[1] ANTUNES, L. E .C. Amora-preta: Nova opção de cultivo no Brasil. Ciência Rural, Santa Maria, v. 32, n. 1, p. 151-158, 2002.

[2] ANTUnES, L. E. C.; DUARTE FILHO, J.; SOUZA, C. M. Conservação pós-colheita de frutos de amoreira-preta. Pesquisa Agropecuária Brasileira, Brasília, v. 38, n. 3, p. 413-419, 2003.

[3] ANTUNES, L. E. C.; REGINA, M. A.; DUARTE FILHO, J. A cultura da amora-preta. Belo Horizonte: EPAMIG, 2002. 28p. (EPAMIG. Boletim Técnico, 69).

[4] BOBBIO, F. O.; BOBBIO, P. A. Pigmentos naturais. In: Introdução à Química de Alimentos. 3. ed. São Paulo: Livraria Varela, 2003. Cap. 6, p. 205-238

[5] CARDOSO, R. L.; FERREIRA, V. L. P.; MONTGOMERY, M. W.; YOTSUYANAGI, K. Efeito do tempo, luz e temperatura na cor da geléia de jambo vermelho (Eugenia malaccensis, Lin). Ciência e Tecnologia de Alimentos, Campinas, v. 17, n. 1, p. 28-31, 1997.

[6] CETEC. Manual para fabricação de geléias. Belo Horizonte: CETEC, 1985. Caps. 3 e 4, p. 17-30.

[7] CORDENUNSI, B. R.; NASCIMENTO, J. R. O.; LAJOLO, F. M. Physico-chemical changes related to quality of five strawberry fruit cultivars during cool-storage. Food Chemistry, v. 83, p.167-173, 2003.

[8] DUBOIS, M.; GILES, K. A.; HAMILTON, J. K.; REBERS, P. A.; SMITH, F. Colorimetric method for determination of sugars and related substances. Analytical Chemistry, v. 28, p. 350, 1956.

[9] ESTAT - SISTEMA PARA ANÁLISES ESTATÍSTICAS. Pólo computacional/Departamento de Ciências Exatas. UNESP-FCAV - Campus de Jaboticabal. V. 2.0.

[10] FOLHA DE SÃO PAULO. Suco de amora ajuda a combater doenças do coração, diz estudo. Edição de 25/03/03. In: TODA FRUTA. Curiosidades Gerais. Disponível em: < http://www.todafruta.com.br $>$. Acesso em: 7 jan. 2005.

[11] FREEDMAN, L.; FRANCIS, F. J. Effect of ascorbic acid on color of jellies. Journal of Food Science, v. 49, p. 1212-1213, 1984.

[12] GARCIA-ALONSO, F. J.; PERIAGO, M. J.; VIDALGUEVARA, M. L.; CANTOS, E.; ROS, G.; FERRERES, F.; ABELLAN, P. Assessment of the antioxidant properties during storage of a dessert made from grape, cherry and berries. Journal of Food Science, v. 68 , n. 4, p. 1525-1530, 2003. 
[13] GLOBO REPORTER. Mirtilo, o blueberry brasileiro. Edição de 29/03/04. In: TODA FRUTA. O poder de cura das frutas. Disponível em: http://www.todafruta. com.br. Acesso em: 11 jan. 2005.

[14] HASsimotTo, N. M. A.; GOMEZ, M. L. P. A.; MOTA, R. V.; CORDENUNSI, B. R.; LAJOLO, F. M. Compostos antioxidantes da amora-preta (Rubus sp.). In: CONGRESSO BRASILEIRO DE CIÊNCIA E TECNOLOGIA DE ALIMENTOS, XIX, 2004, Recife, Resumos... Resumo expandido n. 424, cd-room

[15] INSTITUTO ADOLFO LUTZ. Normas Analíticas do Instituto Adolfo Lutz. Métodos químicos e físicos para análise de alimentos. V. 1, 3. ed. São Paulo: Inst. Adolfo Lutz, 1985. 533 p.

[16] LIMA, V. L. A. G.; MELO, E. A.; LIMA, D. E. S. Efeito da luz e da temperatura de congelamento sobre a estabilidade das antocianinas da pitanga roxa. Ciência e Tecnologia de Alimentos, Campinas, v. 25, n. 1, p. 92-94, 2005.

[17] LIMA, V. L. A. G.; MELO, E. A.; MACIEL, M. I. S.; LIMA, D. E. S. Avaliação do teor de antocianinas em polpa de acerola congelada proveniente de frutos de 12 diferentes aceroleiras (Malpighia emarginata D. C.). Ciência e Tecnologia de Alimentos, Campinas, v. 23, n. 1, p. 101-103, 2003.

[18] MARKAKIS, P; LIVINGSTON, G. E.; FELLERS, C. R. Quantitative aspects of strawberry pigment degradation. Food Research, v. 22, p. 117-130, 1957.

[19] MORENO-ALVAREZ, M. J.; MATOS, A. V.; LÓPEZ, E., BELÉN, D. Estabilidad de antocianinas em jugos pasteurizados de mora (Rubus glaucus Benth). ALAN, v. 52, n. 2, supl. 2, 2002, online.

[20] PERKINS-VEAZIE, P.; CLARK, J. R.; HUBER, D. J.; BALDWIN, E. A. Ripening physiology in 'Navaho' thornless blackberries: color, respiration, ethylene production, softening, and compositional changes. Journal of the American Society for Horticultural Science, v. 125, n. 3, p.357-363, 2000.

[21] PERKINS-VEAZIE, P.; COLLINS, J. K. Quality of erecttype blackberry fruit after short intervals of controlled atmosphere storage. Postharvest Biology and Technology, v. 25, p. 235-239, 2002.

[22] WANG, S. Y.; LIN, H. S. Antioxidant activity in fruits and leaves of blackberry, raspberry, and strawberry varies with cultivar and developmental stage. Journal of Agricultural and Food Chemistry, v. 48, n. 2, p. 140-146, 2000.

[23] ZAFRILlA, P.; FERRERES, F.; TOMÁS-BARBERÁN, F. A. Effect of processing and storage on the antioxidant ellagic acid derivatives and flavonoids of Red Raspberry (Rubus idaeus) Jams. Journal of Agricultural and Food Chemistry, v. 49, p. 3651-3655, 2001.

\section{6 - AGRADECIMENTOS}

Ao CNPq pela Bolsa de Recém Doutor.

Aos funcionários e pesquisadores da Estação Experimental de Caldas (EPAMIG/FECD) que proporcionaram os meios necessários para a realização deste trabalho. 